\title{
EXTENDED CLASSICAL MODELS FOR MULTIPLE CHARGE EXCHANGE
}

\author{
A. NIEHAUS

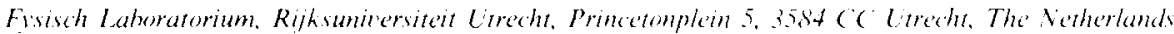

The essential features of a recently developed classical model for multiple electron exchange in collisions of highly charged ions with atoms are outlined. Several extensions of the model are discussed and tented by comparison with experimental data. In most cases it is found that the predictions of these models are strprisingly realistic in spite of their great simplicity. It is concluded that the classical mechanism on which the models are based is essentially correct.

\section{Introduction}

Experimental data concerning charge exchange processes in collisions of highly charged ions with atoms

$\mathrm{A}^{q+}+\mathrm{B} \rightarrow A^{(q)}+\mathrm{B}^{r+}$,

have shown that the corresponding total cross sections are virtually independent of the collision velocity within the range where the velocity of the bound electrons is larger than the collision velocity. This suggests that one might be able to describe the main physics involved in terms of a "static" model. Such a model has already been proven to be rather good for single electron capture [1]. An extension of this so called "classical over barrier" model to multiple electron capture has recently been formulated by Bárány et al. [2]. This extended model allows one to predict absolute cross sections for target loss of $(r)$ electrons, but does neither specify for the different ways in which these $(r)$ electrons may be captured, nor does it predict final electronic states of target and projectile. Nevertheless, comparison with experimental absolute total cross sections 9 for the systems $\operatorname{Ar}^{q^{+}} / \operatorname{Ar}(q=4-8)[2]$ gave rather satisfactory agreement. The models which will be discussed in this paper are also based on the application of the "overbarrier criterion", but are much more detailed. Since they are partly described elsewhere [3,4] only the essential features are outlined here. The main emphasis in this paper is put on a confrontation of the models with experimental data, and on a discussion of further modifications.

\section{The model}

The main idea - in contrast to the earlier overbarrier models - is that capture does not occur "on the way in" when the collision partners approach, but rather "on the way out", when they separate. On the way in, a certain number of electrons originally bound to the target become "molecular", because the Coulomb barrier between target and projectile successively ceases to be effective for these electrons at critical distances $R_{1}^{\prime}$. $R_{2}^{\prime}, \ldots R_{3}^{\prime} \ldots$ On the way out then, these molecular electrons may be captured with certain probabilities by projectile or target. This capturing is caused by the rising Coulomb barrier which separates the space accessible to the electroms at critical distances $R_{i}^{\prime \prime}$. By applying the overbarrier criterion for each target electron. it follows that the molecular binding energies $E_{1}, E_{2}, \ldots$ $E_{r} \ldots$, are ordered in the same way as the target ionization energies, $I_{1}, I_{2}, \ldots I_{t} \ldots$, namely $R_{1}^{0}>R_{1}^{(1,1}$, etc. To calculate a certain critical distance $R_{1}^{11}$ from the criterion that the Coulomb barrier height is equal to the binding energy $E_{\mathrm{t}}$, it is necessary to specify for the number of electrons $r_{t},\left(t^{\prime}>t\right)$ that have been captured by the projectile at smaller distances $R_{t}^{0}$. By neglecting the screening of "outer electrons" thruughout the collision. the distances $R_{t}^{i}$ and $R_{t}^{0}\left(r_{t}\right)$ can be calculated for a charge changing collision specified by the numbers $r_{t}$. i.e. by defining where each electron finally is captured. To characterize such a well defined process, we use a string $(j)$ in which the position indicates the index $(t)$ and a " 1 " indicates capture by the projectile while a " 0 " indicates recapture by the target. A process with string. $(j)=(10100000)$,

for instance, would indicate a process where the target clectrons with ionization potentials $I_{1}$ and $I_{3}$ are captured by the projectile, while the others of the 8 electrons considered are recaptured or remain "atomic" throughout the collision.

In order to contribute to the example process (2), the turning point of a collision ( $R_{\mathrm{TP}}$ ) has to be smaller than $R_{3}^{\prime}$, the distance at which the third electron becomes molecular on the "way in". For collisions with turning points $R_{\mathrm{TP}}<R_{t}^{\prime}\left(t \geqslant t_{0}=3\right)$ the probability for process (2) to occur depends on $t$. Calling this probability $P_{t}^{(\prime)}$, 
we can express the cross section in the straight line approximation generally as

$\sigma_{y, y,}^{\prime \prime \prime},=\sum_{t=t_{1}}^{x} \pi\left\{\left(R_{t}^{\prime}\right)^{2}-\left(R_{t, 1}^{\prime}\right)^{2}\right\} P_{t}^{\prime \prime \prime}$.

where $x$ is the maximum number of electrons that can become molecular. The probabilities $P_{r}^{(1)}$ are products of the appropriate single electron probabilities. These single electron probabilities are the crucial quantitics of the model. Quantum mechanically one would estimate these probabilities from a projection of the molecular orbital at $R_{f}^{\prime \prime}\left(r_{t}\right)$ onto atomic orbitals centered around projection and target. Since it was the aim to keep the simplicity of the model, in the original version the degree of degeneracy of the possible Coulomb-orbitals on projectile and target was used to estimate the capture probability. Calling $n(t)$ and $m(t)$ the principal quantum numbers of the corresponding orbitals - i.e. of those orbitals into which the electron would be captured after separation -.- the single electron capture probabilities are simply given by

$H_{t}^{\prime \prime \prime}=n(t)^{2} /\left(n(t)^{2}+m(t)^{2}\right)$.

where the quantum numbers are assumed to be continuous. In Coulomb approximation these numbers are obtained from the atomic binding energies, which within the model are given by

$$
\begin{aligned}
& \mathrm{EP}_{l}^{\prime \prime \prime}=I_{t}+q / R_{t}^{i}-\frac{t+r_{t}}{R_{t}^{\prime \prime}\left(r_{t}\right)} \\
& \mathrm{ET}_{r}^{\prime \prime \prime}=I_{t}-q / R_{t}^{\prime}-\frac{q^{-r_{t}}}{R_{t}^{\prime \prime}\left(r_{t}\right)} .
\end{aligned}
$$

for capture by the projectile, and by the target, respectively. In addition to the cross sections $\sigma_{q, q}^{(j)}$, . also the kinetic energy gain can be calculated from the binding energies, eq. (5). Denoting the actual binding energy of the $(t)$ th electron in a process $(j)$ by $\epsilon_{i}^{(j)}$, where

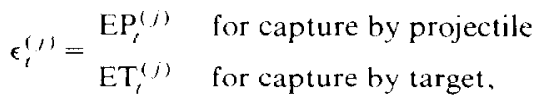

the energy gain is simply

$Q^{\prime \prime \prime}=\sum_{i=1}^{t_{1 \prime}}\left(\epsilon_{i}^{\prime \prime}-I_{t}\right)$.

\section{Discussion}

The model predicts absolute cross sections for capture processes. When the predictions are to he compared to experimental data, one has to consider possible decay processes of the initially formed atoms, and further, one has to sum over all theoretical cross sections which are not distinguished in the experiment. Tawara et al. [5] have studied collisions of $\mathrm{I}^{\text {" }}$ " with $\mathrm{He}$ at low collision energies for $q-1041$. They determined absolute cross sections for the formation of $I^{\prime /} 11$. Since double capture is followed by autoionization for higher q. the measured cross section $\sigma_{q}$, , contains all three theoretical cross sections $\sigma_{q}^{(10)}, \sigma_{q}^{(1)}$ and $\sigma_{q}^{(1)}$. In fig. 1 these cross sections are shown together with their sum, which should be compared to the experimental points taken from the work of Tawara et al. [5]. The agreement is rather good. This is also true for the average principal quantum number predicted by the model.

The advantage of the simplicity of the model is that predictions can be made also for complicated systems. Although we do not expect the predictions to be always as accurate as in the case of $I^{*} / \mathrm{He}$, we believe that the relative importance of the vast number of different charge changing processes is probably rather realistically estimated. In fig. 2 we show in which way for the system $\mathrm{Ar}^{4}$. $\mathrm{Ar}$ the "geometrical cross section" $\sigma_{\text {a.w. }}$ $=\pi R_{1}^{2}$ is divided up into the partial cross sections $\sigma^{\prime \prime \prime \prime) . ~}$ For the capture of $(r)$ electrons there are $\left({ }^{\prime}\right)$ partial cross sections $\sigma_{4}^{(\prime)}$ as indicated by the strings $(j)$ in fig. 2. For each individual process the binding energy of

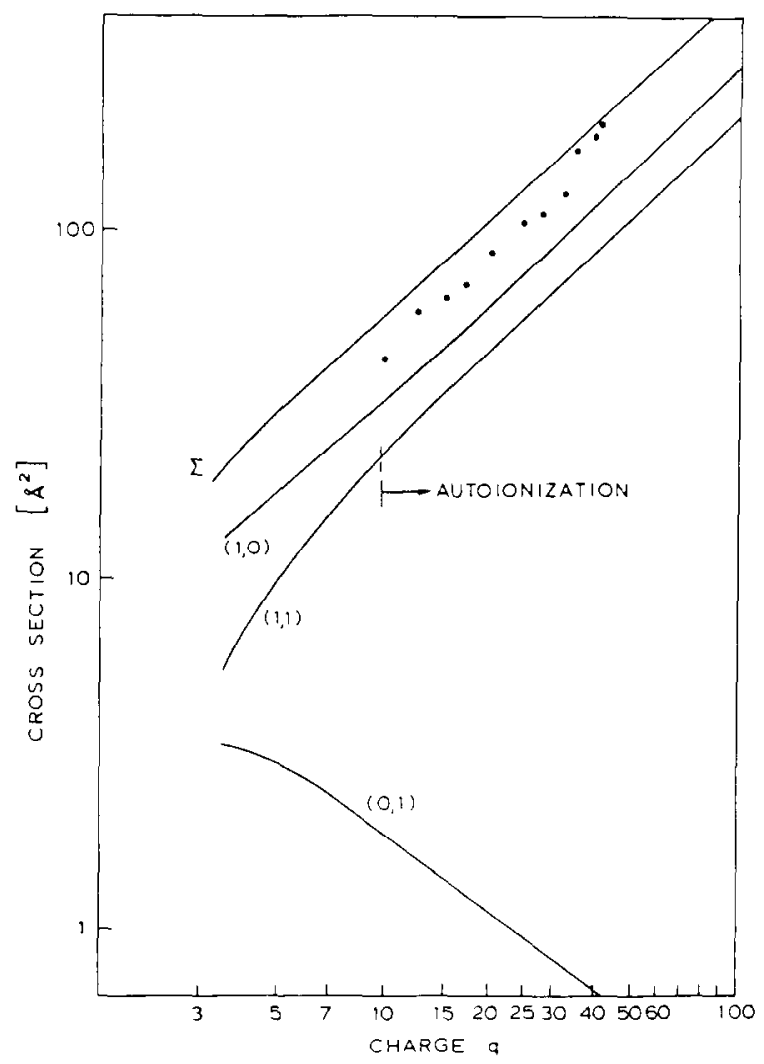

F̈ig. 1. Model cross sections for single capture (1. (0) and (0. 1). and double capture $(1,1)$ in $1^{\%} /$ He-collisions. The sum ( $\left.\Sigma\right)$ of these cross sections is compared to the experimental cross section for the formation of $\mathrm{I}^{1 / 2} 11+$ (e) $|5|$. 
each electron is predicted. This allows one to predict whether the process leads to decay by electron emission. Since the binding energy of the electrons generally decreases when $q$ increases, there arise limits on the $q$-scale beyond which a certain process is followed by autoionization. A few such limits for the case of projectile autoionization are indicated in fig. 2. Target autoionization is expected for $q \geqslant 5$ following the capture of two or three "inner" electrons by the projectile. Generally, the prediction is that projectile autoionization by far predominates.

For collisions of $\mathrm{Ar}^{4}$ " with Ar Astner et al. [6] have obtained cross sections for the formation of projectiles of charge ( $k$ ) and target ions of charge ( $s$ ), using a coincidence technique. These cross sections $\sigma_{c}^{\prime}$ h are composed of several partial cross sections $\sigma_{q}^{\prime \prime \prime}$. In table
1 we show the relation between the $\sigma_{q}^{(j)}$ and the $\sigma_{q . k}^{*}$ with the experimental values. From the rather good agreement of all the numbers we conclude that even in complicated systems the main mechanism of charge exchange is well described by the model.

From measurements of the gain of kinetic energy $Q$ in charge changing collisions $[7,8]$, as well as from measurements of autoionization electrons [9] and of photons [10] it is well known, that states lying within an cnergy window of a certain width are populated in a capture process, while the model described so far predicts a sharp binding energy. This sharp value probably has to be interpreted as an average value. By comparing the widths of the windows found experimentally, we noticed that they tend to be larger at larger collision velocity. This indicates a dynamic origin. Some dynamic

Iable 1

Comparison of experimental cross sections (last column [6]) with model cross sections (last but one column) for the phenomenological processes $q \rightarrow k, s$ in $\mathrm{Ar}^{5+}+\mathrm{Ar} \rightarrow \mathrm{Ar}^{k+}+\mathrm{Ar}^{5+}$. In the first column the string $(j)$ characterizing the different processes distinguished in the model is given. The second column gives the absolute model cross section, and in the third column it is indicated whether or not autoionization is predicted for target or projectile. In the fourth column the phenomenological process to which a process of defined string ( $j$ ) contributes is indicated

\begin{tabular}{|c|c|c|c|c|c|c|}
\hline \multirow{2}{*}{$\begin{array}{l}\text { Process }(j) \\
t=12345\end{array}$} & \multirow{2}{*}{$\begin{array}{l}\sigma^{(j)} \\
{\left[\stackrel{\mathrm{A}}{2}^{2}\right]}\end{array}$} & \multirow{2}{*}{$\begin{array}{l}\text { Auto- } \\
\text { ionization }\end{array}$} & \multicolumn{2}{|c|}{ Phenomenological processes } & \multirow{2}{*}{$\begin{array}{l}\text { Model } \\
\text { cross section } \\
\sigma\left[\tilde{A}^{2}\right]\end{array}$} & \multirow{2}{*}{$\begin{array}{l}\text { Experimental } \\
\text { cross section } \\
\sigma\left[\AA^{2}\right]\end{array}$} \\
\hline & & & $q \rightarrow k, s$ & $q \rightarrow k, s$ & & \\
\hline 10000 & 17.6 & - & $5 \rightarrow 4.1$ & & & \\
\hline 01000 & 6.9 & - & $5 \rightarrow 4,1$ & $5 \rightarrow 4,1$ & 28 & 26 \\
\hline 00100 & 2.4 & - & $5 \rightarrow 4.1$ & & & \\
\hline 00010 & 0.8 & - & $5 \rightarrow 4,1$ & & & \\
\hline 00001 & 0.6 & - & $5 \rightarrow 4.1$ & & & \\
\hline 11000 & 10.9 & proj. & $5 \rightarrow 4,2$ & $5 \rightarrow 4,2$ & 10.9 & 7.8 \\
\hline 10100 & 3.6 & - & $5 \rightarrow 3,2$ & & & \\
\hline 10010 & 1.3 & - & $5 \rightarrow 3,2$ & & & \\
\hline 10001 & 0.9 & - & $5 \rightarrow 3.2$ & & & \\
\hline 01100 & 3.6 & - & $5 \rightarrow 3.2$ & $5 \rightarrow 3.2$ & 11.7 & 9.2 \\
\hline 01010 & 1.3 & - & $5 \rightarrow 3.2$ & & & \\
\hline 01001 & 1.0 & - & $5 \rightarrow 3.2$ & & & \\
\hline 00110 & 1.3 & target & $5 \rightarrow 3.3$ & & & \\
\hline 00101 & 1.0 & target & $5 \rightarrow 3,3$ & & & \\
\hline \multirow[t]{2}{*}{00011} & 1.0 & target & $5 \rightarrow 3,3$ & & & \\
\hline & & & & $5 \rightarrow 3,3$ & 9.2 & 8.4 \\
\hline 11100 & 3.6 & proj. & $5 \rightarrow 3.3$ & & & \\
\hline 11010 & 1.3 & proj. & $5 \rightarrow 3,3$ & & & \\
\hline 11001 & 1.0 & proj. & $5 \rightarrow 3.3$ & & & \\
\hline 10110 & 1.3 & - & $5 \rightarrow 2,3$ & & & \\
\hline 10101 & 1.0 & - & $5 \rightarrow 2,3$ & $5 \rightarrow 2,3$ & 5.6 & 4.6 \\
\hline 10011 & 1.0 & - & $5 \rightarrow 2.3$ & & & \\
\hline 01110 & 1.3 & - & $5 \rightarrow 2.3$ & & & \\
\hline 01101 & 1.0 & - & $5 \rightarrow 2,3$ & & & \\
\hline 01011 & 1.0 & -- & $5 \rightarrow 2,3$ & & & \\
\hline 00111 & 1.0 & target & $5 \rightarrow 2.4$ & $5 \rightarrow 2.4$ & 1.0 & 0.9 \\
\hline 11110 & 0.8 & - & $5 \rightarrow 1,4$ & & & \\
\hline 11101 & 0.6 & - & $5 \rightarrow 1,4$ & & & \\
\hline 11011 & 0.6 & - & $5 \rightarrow 1,4$ & $5 \rightarrow 1,4$ & 3.0 & - \\
\hline 10111 & 8.7 & - & $5 \rightarrow 1,4$ & & & \\
\hline 01111 & 8.3 & - & $5 \rightarrow 1,4$ & & & \\
\hline
\end{tabular}




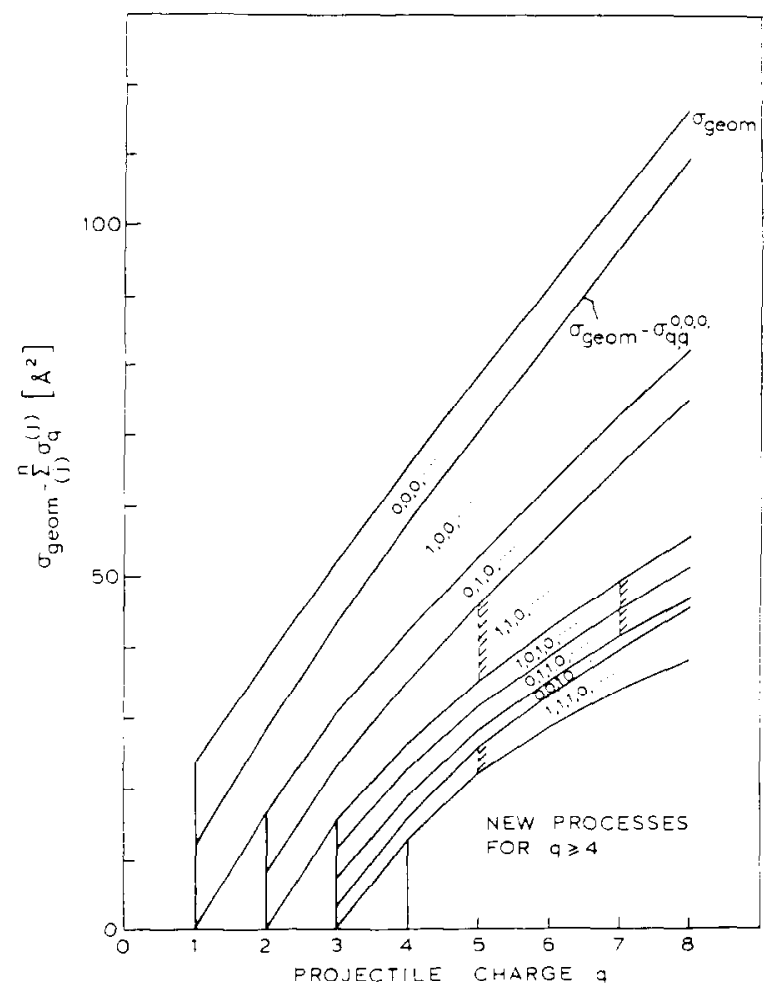

Fig. 2. Calculated composition of the geometrical cross section $\sigma_{\mathrm{geom}}=\pi\left(R_{1}^{i}\right)^{2}$ by the individual cross sections $\sigma_{4}^{(\prime \prime}$ indicated by the corresponding strings, for the systems $\mathrm{Ar}^{q 4} / \mathrm{Ar}$. Predicted limits for autoionization are also indicated.

behaviour can be incorporated into the "static model" described so far, by taking into account that the overbarrier criterion is uncertain due to the time variation of the barrier height. We introduced a "minimum uncertainty" of the barricr height which is given by

$\Delta V_{\mathrm{b}}=\sqrt{\nu_{\text {ract }} v_{\mathrm{h}}}$.

with $\nu_{\text {rad }}$ the radial velocity and $V_{b}^{\prime}$ the derivative of the barrier height with respect to the distance between the collision partners. In this way the binding encrgies $E_{r}^{\prime \prime}$ defined in eq. (6) are replaced by a Gaussian distribution of binding energies, and, correspondingly, the energy gain $Q$ by a distribution of energy gains. The widths of the distributions corresponding to different indices $(t)$ are assumed to add up quadratically.

In figs. 3 and 4 the Gaussian distributions on the $Q$-scale calculated for the processes $j \equiv(10),(01),(11)$ in collisions of $\mathrm{Ar}^{6+}$ and $\mathrm{Ne}^{7+}$ with $\mathrm{D}_{2}$, are compared with experimental projectile energy gain spectra for single capture. For the very small scattering angles arising in these processes, and for the collision energy which corresponds to a relative energy that is large compared to the relevant $Q$-values, the projectile energy gain-value differs from the corresponding $Q$-value by an insignificant amount. The experimental spectra con-

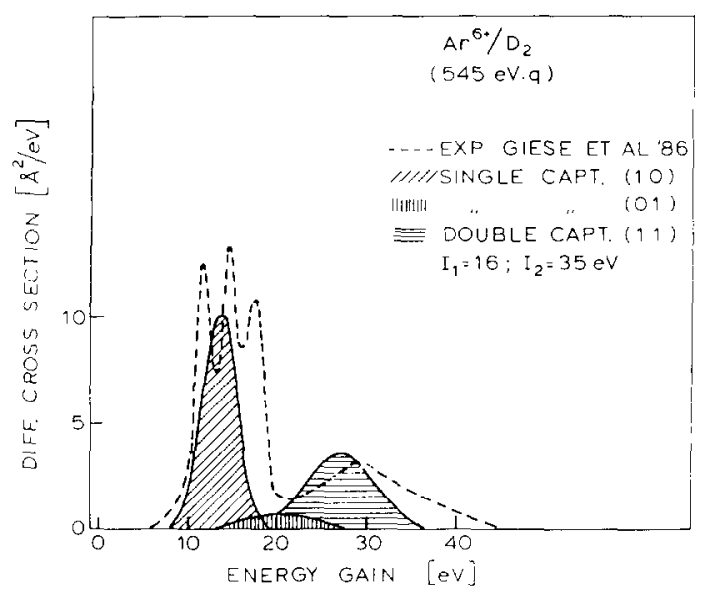

Fig, 3. Experimental energy gain spectrum for $\mathrm{Ar}^{5}$. Formed in $\Delta r^{6} / D_{2}$ collisions (.., $[8]$, and calculated "Galussians" for the processes indicated by the strings. The absolute cross section scale relates to the Gaussians.

tain contributions from pure single capture, and from double capture followed by autoionization [8]. Width and position of the theoretical spectra agrec qualitatively with the experimental ones. The two examples indicale in which way the theoretical " $Q$-window" should be interpreted: the area under the Gaussians corresponds to the maximum possible cross section, which is realized only if states are available in the relevant energy range. Following this interpretation the predicted cross sections are lower for $\mathrm{Ne}^{7} / \mathrm{D}$, than for $\mathrm{Ar}^{h} / \mathrm{D}_{2}$, as indicated. In fig. 5 autoionization electron spectra following double capture are shown [11]. These spectra are mainly due to population of $\left(3 \ln ^{\prime} l^{\prime}\right)$-states which decay to the (2pe)-continuum. The electron en-

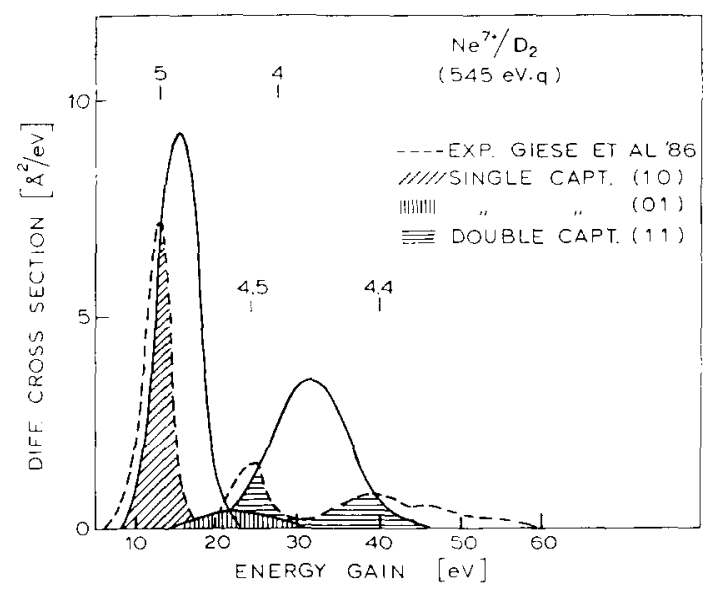

Fig. 4. Experimental energy gain spectrum for $\mathrm{Ne}^{\text {6 }}$ formed in $\mathrm{Nc}^{7}+\mathrm{D}$ z collisions (....,$[\mathrm{x}]$, and calculated "Gaussians" for the processes indicated by the strings. The absolute cross section scale relates to the Gaussians. 

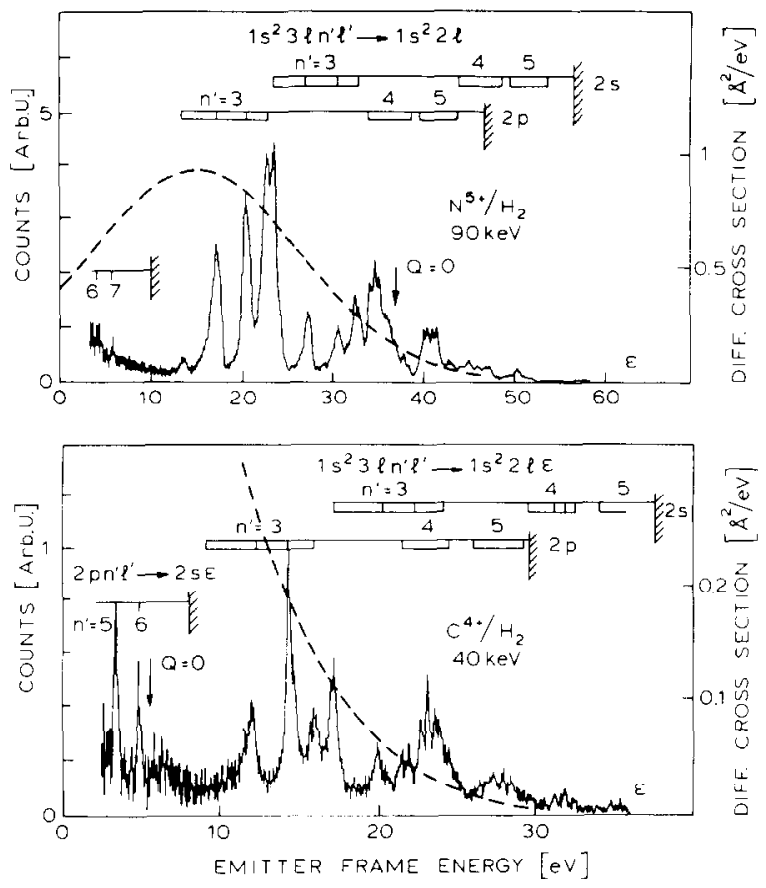

Fig. 5. Autoionization electron energy spectra for $\mathrm{N}^{5} / \mathrm{H}_{2-}$ and $\mathrm{C}^{4+} / \mathrm{H}_{2}$-collisions. Also indicated the predicted "Gaussians". The absolute cross section scale relates to the (ratussians. The count rate seales are the same for the two experimental spectra.

ergy "window" corresponding to this decay and to the calculated population window is indicated. The intensity scales for the two experimental spectra are comparable, and normalized to the absolute differential cross section scale by comparison with the theoretical Gaussians. This nomalization is somewhat asbitrary, however, the low cross section observed for $\mathrm{C}^{+} / \mathrm{H}_{2}$ is well explained by the fact that states are onlv available in the wings of the population window. The position, the width, and the velocity dependence of the width of the predicted population windows have been compared for several systems with measured autoionization spectra [11]. In all cases good qualitative agreement is observed.

The introduction of an energy dependent population window implics that the model in the extended form predicts the energy dependence of absolute partial cross sections. This is also true for endothermic processes, since the model does not distinguish between endothermic and exothermic processes. For some endothermic processes causing HcII-line cmission in $Z^{\prime \prime} / \mathrm{Hc}$ collisions $(Z=C, N . O$. Ne, $\operatorname{Ar} ; q=4,5)$ a quantitative comparison with experimental data was performed [12], and reasonable agrecment in both magnitude and impact velocity dependence was found.

The model, so far, does not account for an influence

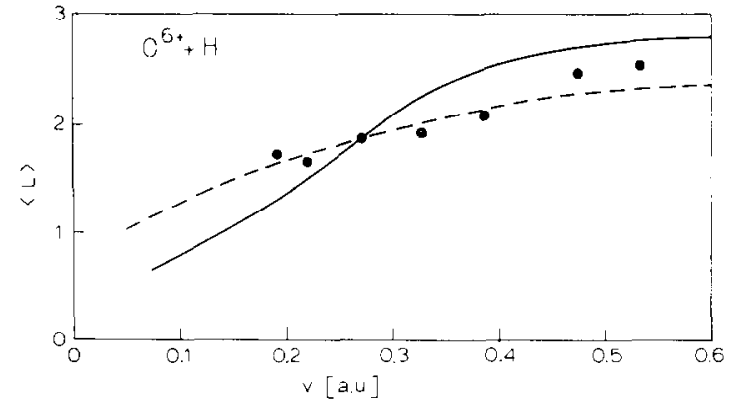

Fig. 6. Variation of the average angular momentum of the $\mathrm{O}^{5+}\left(1 \mathrm{~s}^{2} 4 \mathrm{~L}\right)$ states formed in $\mathrm{O}^{6+} / \mathrm{H}_{2}$-collisions. (め) experiments [10]; (steep curve) model [4]; (other curve) modified model [13].

of the orbital angular momentum of a state on its population in a capture process. For the case of single capture recently a first attempt has been made to introduce such an influence by assuming that the electronic angular momentum, caused by the relative motion of the heavy particles, is conserved and appears as orbital angular momentum in the state populated by capture [4]. In this way only states with angular momenta $L<V R_{i}^{i}(L)$ can be populated, with $V$ the relative collision velucity and $R_{t}^{\prime}(L)$ the critical overbarrier distance

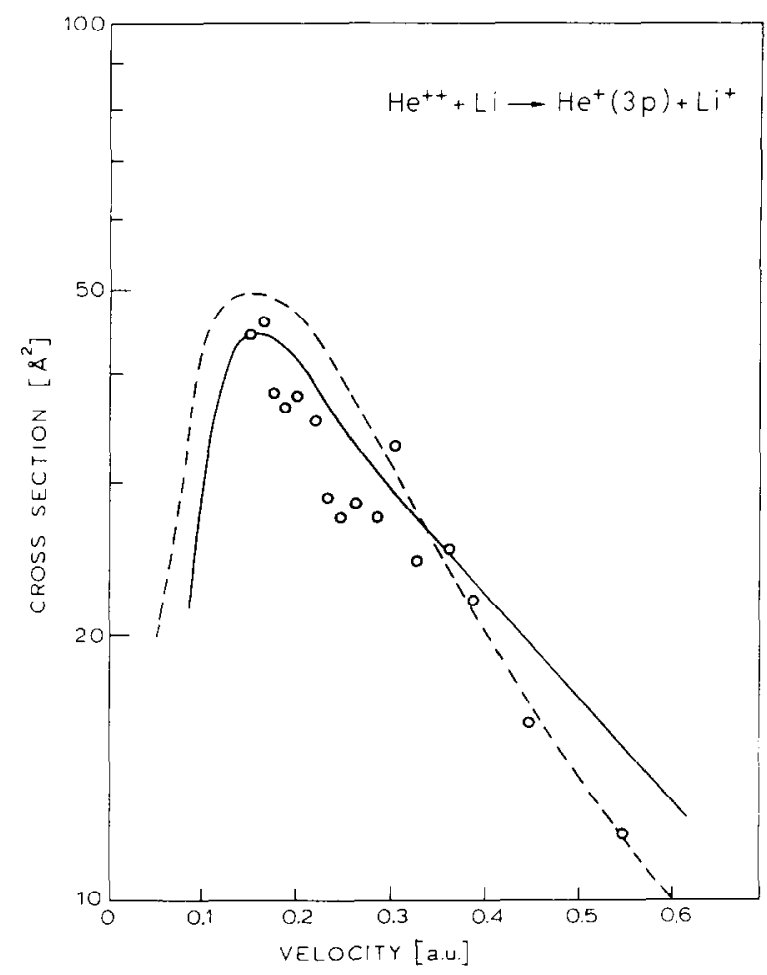

Fig. 7. Absolute subshell cross section $\sigma_{3 p}$ for the indicated process. (100) experiment [15]; (-) theory [14] (, ) model. 
whose dependence on $L$ is also accounted for. While this approach is certainly much too simple to describe the observed [10] strong and seemingly irregular variations of the subshell cross sections $\sigma_{n /}$. it might be appropriate to describe the average value of the angular momentum $\langle L\rangle$ within a shell. For several systems $\mathrm{A}^{4}+/ \mathrm{H}, \mathrm{H}_{2}$ a comparison of the velocity dependence of the calculated $(L)$ with experimental data of Dijkkamp et al. [10] has been made [4]. As an example we show in fig. 6 the result for the population of $(n=4)$-states by single capture in $\mathrm{O}^{6} / \mathrm{H}$ collisions. Also shown is the result of a somewhat improved description [13] which allows for a certain width $\Delta L$ of the angular momentum carried into the electronic state via the heavy particle motion. In all cases studied, the smooth increase of $\langle L\rangle$ with collision velocity is well described by the model.

As already mentioned, many of the observed subshell cross sections [10] show strong and seemingly unsystematic variations with impact velocity which cannot be described by the present model. However, there are also examples where the $\sigma_{n}$, vary rather smoothly, probably indicating that some couplings usually present among the different $L$-states are absent. In these cases the observed variation of $\sigma_{n /}$ is rather well described by the simple model. An example is shown in fig. 7 for the $\mathrm{He}^{1}(3 p)$-state populated by capture in collisions of $\mathrm{He}^{2}$ ' with $\mathrm{Li}$.

\section{Conclusions}

From the confrontation of the predictions of the classical model with the available experimental data. we conclude that the model is realistic in the sense that it formulates classically the main mechanism of multiple electron exchange in slow collisions of highly charged ions with atoms. Since a more rigorous theory is not avaible for these many-electron processes, the model is valuable because it affords a rather straight forward physical interpretation of experimental data, and allows one to make rather realistic quantitative predictions, even for systems of high complexity. In some cases the predictions need appropriate interpretation in order to be realistic. Improvements of the model as described here are necessary and seem possible. Expecially, the crucial quantity of the model, the single electron capture probability, should be modified to incorporate the angular momentum restrictions which arise due to the at least partial - conservation of the electronic angular momentum carried by the captured electron due to the heavy particle motion.

\section{References}

[1] H. Ryufuku, K. Sasaki and T. Watanabe, Phys. Rev. A21 (1980) 745 .

[2] A. Bárány, G. Astner, H. Cederquist, H. Danared, S. Huldı, P. Hvelplund, A. Johnson, H. Knudsen, L. Liljeby and K-G. Rensfelt, Nucl. Instr. and Meth. B9 (1985) 397.

[3] A. Niehaus, J. Phys. B19 (1986).

[4] J. Burgdüıfer, R. Morgenstern and A. Nielıaus. J. Plıys B19 (1986) L507.

[5] H. Tawara, T. Iwai, Y. Kaneko, M. Kimura, N. Kobayashi, A. Matsumoto, S. Ohtani, K. Okuno, S. Tagaki and S. Tsurubuchi, J. Phys. B18 (1985) 337.

[6] G. Astner, A. Bárány, H. Cederquist. H. Danared, S. Huldt, P. Hvelplund, A. Johnson, H. Knudsen, L. Lijjeby and K-G. Rensfelt, J. Phys. B17 (1984) L877.

[7] F.H. Nielsen, I.H. Andersen, A. Bárány, H. Cederquist, P. Hvelplund. H. Knudsen, K.B. McAdam and J. Sorensen, J. Phys. B17 (1984) L139.

[8] J.P. Giese, C.L. Cocke, W. Waggoner, L.N. Tunnell and S.L. Varghese (1986) private communication.

[9] A. Bordenave-Montesquieu, P. Benoit-Cattin, A. Gleizes, A.I. Marakchi, S. Dousson and D. Hitz, J. Phys. B17 (1984) L223.

[10] D. Dijkkamp, A. Brazuk, A.G. Drentje. F.J. de Heer and H. Winter, J. Phys. B17 (1984) 4371.

[11] M. Mack and A. Niehaus, these Proceedings (Conf. on the Physics of Multipy Charged lons, Groningen, The Netherlands, 1986) Nucl. Instr. Meth. B23 (1987) 109/116/291.

[12] R. Hoekstra. F.J. de Heer and H. Winter, these Proceedings (Conf. on the Physics of Multiple Charged Ions, Groningen. The Netherlands, 1986) Nucl. Instr. and Meth. B23 (1987) 104.

[13] J. Burgdörfer, K. Morgenstern and A. Niehaus ibıdem, p 120.

[14] W. Fritsch and C.D. Lin, J. Phys. B16 (1983) 1595.

[15] D. Dijkkamp, Thesis, Rijksuniversiteit Utrecht (1985). 